latter is a more convenient method with adult Ss, who may also be asked to fixate points of known location in the room while keeping the head immobile.

Because of the early maturation of accurate compensatory adjustments, $^{6}$ a record of 1 or 2 min of spontaneous visual exploration of a stationary array, occupying at least 90 deg of the field, is generally sufficient for accurate matching of head and eye signals with an attentive $S$ over the age of 2 months. When this adjustment is correctly made, the summed record of gaze displacement shows almost complete compensation for head rotations in both directions, and the portions of the trace between eye saccades are flat (Fig. 3). Consistent deviations indicate that the gain for the EOG is too high or too low.

The outputs of the amplifiers for the EOG and for both bridges are connected through an appropriate switching circuit to an electronic sum and difference circuit. ${ }^{7}$ The switching at the input to these analog devices is arranged so that any of the signals can be added to or subtracted from any other signal or from any combination of signals (Fig. 2B). The output of these networks is displayed on separate channels of the polygraph. Thus, the angle of the direction of gaze in the horizontal plane is the sum of the head and eye voltage, and the difference between this sum and the target voltage is the tracking error (Fig. 3).

\section{DIRECTION-OF-GAZE SWITCH FOR THE CONTROL OF VISUAL EXPLORATION}

The dc "line-of-sight" signal has been employed to regulate a stimulus. An angle of visual space is designated, and the stimulus may be made to appear only when the $S$ is looking outside it. Electronic level detectors have been used to delineate the width of the field, the boundaries of which may be adjusted independently to obtain an "on" or "off" zone of any width in any part of the field. In other words, it is possible to arrange for a stimulus to appear only at a designated locus in the visual field or in a large or small part of the field.

We have applied this method for verification of fixation while testing peripheral vision and in the study of infant vision.

\section{REFERENCES}

MARG, E. Development of electro-oculography: Standing potential of the eye in registration of eye movement. Archives of Opthalmology, 1951, $45,169-185$

O'CONNELL, D. N., \& TURSKY, B. Silver-silver chloride sponge electrodes for skin potential recording. American Journal of Psychology, 1960, 73, 302-304.

YOUNG, L. Measuring eye movements. American Joumal of Medical Electronics, 1963, 2, 300-307.

\section{NOTES}

1. Support for this work was obtained from the National Institute of Child Health and Development, Grant R01-HD-03049. The apparatus described was developed for research with infants while the senior author was a Research Fellow at the Center for Cognitive Studies of Harvard University. The study of the early development of visual orientation was carried out in collaboration with Dr. J. S. Bruner, with whom the needs of the recording mechanism were determined.

2. Now at the Biology Division, California Institute of Technology, Pasadena, California 91109.

3. We wish to thank Mr. Ralph Gerbrands for his design and fabrication of this exquisite piece of equipment.

4. Giannini Controls Corporation; Microtorque Potentiometer, Model 85111, resistance of 5,000 ohms.

5. Other considerations applying specially to work with young infants will be discussed in a forthcoming publication.

6. Trevarthen, C. 000000000000000000000000000 , in preparation.

7. Mr. James Campbell of the Electrical Engineering Department, Caltech, has helped us in improving the recording circuits.

\title{
A titration procedure for generating escape behavior
}

JAMSHID KHALILI, ${ }^{1}$ MARVIN F. DALEY, and CARL D. CHENEY, UTAH STATE UNIVERSITY, Logan, Utah 84321

In a grid-shock escape paradigm using time-out as reinforcement, both fixed-ratio and variable-interval performances were established directly from a continuous reinforcement schedule with rats and guinea pigs. A procedure was developed that employed a stepwise decrease in reinforcement time for all responses except the terminal response. Acquisition and long-term maintenance data were obtained from Ss trained on this titrated negative-reinforcement procedure.

By far the largest proportion of research on the control and modification of behavior has been accomplished using positive-reinforcement (reward) procedures; i.e., studies using food, intercranial stimulation, or water have predominated. Researchers and theoreticians have realized, however, that much animal behavior in natural as well as in experimental environments is under the control of aversive contingencies. There is an extensive literature on the use of shock to control behavior, although few successful methods for the acquisition and maintenance of extended schedules of escape behavior using grid-delivered electric shock have been devised.

Recently, a technique was reported for developing performance on a ratio schedule using ICS for positive reinforcement (Huston, 1968). It was proposed that fixed-ratio performance could be established directly from a continuous reinforcement regimen by a gradual reduction of the ICS train duration, contingent on all but the terminal response in the ratio.

In light of the difficulties reported in the acquisition and maintenance of grid-escape behavior (Dinsmoor, 1967), we adapted Huston's procedure to escape training, and experiments were conducted to show its reliability, species generalization, and long-term maintenance properties.

In a fixed-ratio (FR) schedule of negative reinforcement, the organism is required to emit a certain number of responses before the final response can produce a time-out or escape from the aversive stimuli. Using rats with subcutaneously implanted electrodes, Es have been unable to obtain or to maintain responding even on small fixed-ratio escape schedules (Hendry \& Hendry, 1963). They reported that ratio requirements as low as 4 showed straining. Winograd (1965) reports a need to stay at FR 1 for as long as 16 sessions before the ratio could be slightly increased. He also found it necessary to shave the bodies of the rats when grid-shock was employed. In spite of these precautions, Winograd observed disruption of behavior during the first few sessions at ratios higher than FR 1 and, in fact, the shock level had to be doubled for one of the Ss in order to maintain any responding. Disorderly records were also obtained using a procedure in which the terminal response of an FR run yielded a 
reduction rather than a termination of shock (Weiss \& Laties, 1959). Schedules of negative reinforcement other than FR have rarely been attempted with animals (Dinsmoor, 1967).

\section{PROCEDURES}

Six adult male guinea pigs and six male Long-Evans rats were used as Ss. All animals had continuous access to food and water in their individual home cages.

All animals were trained and run in the same Grason-Stadler small-animal operant conditioning chamber. A Grason-Stadler Model E1064GS shock generator and scrambler provided shock to the chamber grid, walls, and bar. The operant chamber was housed in a sound-attenuation chamber, equipped with fan and houselight. Standard control equipment, consisting of relays, timers, and cumulative as well as digital recording devices, were used to program and record automatically all experimental contingencies. Sessions were $50 \mathrm{~min}$ long, generally 6 days a week.

The Ss were shaped by successive approximation to press a bar, which resulted in the termination of electric shock delivered through the grid floor. Shock intensity for guinea pigs was $1.0 \mathrm{~mA}$ and for rats $0.6 \mathrm{~mA}$. A continuous reinforcement (CRF) schedule was then employed for one session in which each response produced $30 \mathrm{sec}$ of time-out from shock.

Following this single CRF session, a variety of intermittent reinforcement schedules were employed. Two guinea pigs were put on fixed-ratio (FR) 40, and four others on either variable interval (VI) $60 \mathrm{sec}$ or VI 30 schedules of negative reinforcement. Two rats were put on FR 20, two on FR 40, one on FR 60, and another on FR 80 . The responses meeting the FR or VI contingencies were reinforced with $30 \mathrm{sec}$ of time-out (TO) from shock. All other responses were reinforced with shorter TOs on a CRF basis. These CRF TOs are referred to as titrated reinforcement (TR). Titrated reinforcement was then decreased stepwise, either between (both FR and VI) or within (FR only) sessions, until their values were zero and the behavior was under control of the FR or VI contingencies. A sample series of such TO decreases was FR40TR20 sec, FR40TR15 sec, FR40TR10 sec, FR4OTR5 sec, FR4OTR3 sec, FR40TR1 sec, FR40TR0.5 sec, FR40TR0 sec, which was in fact the primary schedule for two rats. Similar decreases were used in the VI schedules.

\section{RESULTS AND DISCUSSION}

Figure 1, Record A, shows a typical rat performance on FR20TR0 sec. This was the 168th session for Rat No. 2 at these stimulus parameters, although stability was obtained at Session 9 . Record B is a guinea pig on FR40TR0 sec at Session 85. Record C shows a rat on FR60rR0 sec at Session 50. A "warm-up" effect is observed in the first few minutes of the session, but the remainder is consistent and stable. Pausing after reinforcement is comparable to that obtained with a positive fixed-ratio reinforcement procedure (Ferster \& Skinner, 1957). The degree of pausing is a function of the ratio requirement and/or time-out from shock as well as the shock intensity. The pauses in Record C (FR6OTR0) are greater than those observed in A (FR20TR0) or B (FR40TR0), although the reinforcement time and the shock intensity were the same. Record $D$ shows a guinea pig's performance on VI30TR0 sec, Session 70, and Record E shows Session 63 of a guinea pig on VI60TR0 sec.

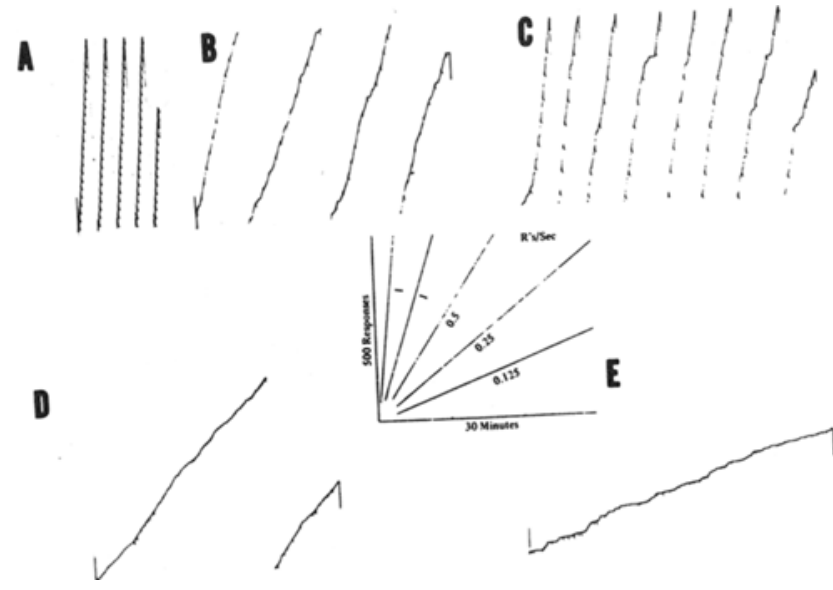

Fig. 1. Cumulative records showing FR and VI grid-shock escape rate of rats ( $A$ and $C$ ) and guinea pigs $(B, D$, and $E$ ). Short downward deflections indicate reinforcement (TO) during which the recorder did not run.

Acquisition for both species was comparable at least on FR schedules. No obvious behavioral differences resulted from titrated reinforcement changes between or within sessions. The final performance for guinea pigs generally shows a less smooth grain than rats. However, this may be due to the different topography of responding and/or the schedule used. Guinea pigs usually pressed with their chins, whereas rats used their forepaws. Long-term maintenance of stable-criterion escape responding as presented here demonstrates that, unlike earlier reports (Winograd, 1965), no body shaving, increases in shock intensity, or long acquisition periods are needed to obtain high rates of shock-escape behavior. In addition, this titrated negative-reinforcement procedure provides a means of establishing escape behavior on schedules directly comparable with those using positive reinforcement.

\section{REFERENCES}

DINSMOOR, J. A. In M. R. Jones (Ed.), Miami Symposium on the Prediction of Behavior: Aversive stimulation. Coral Gables, Fla. University of Miami Press, 1967. Pp. 33-75.

FERSTER, C. B., \& SKINNER, B. F., Schedules of reinforcement. New York: Appleton-Century-Crofts, 1957 .

HENDRY, D. P., \& HENDRY, L. S. Partial negative reinforcement: Fixed-ratio escape. Journal of the Experimental Analysis of Behavior, 1963, 6, 519-523.

HUSTON, J. P. Reinforcement reduction: A method for training ratio behavior. Science, 1968, 159, 444.

WEIS, B., \& LATIES, V. G. Titration behavior on various fractional escape programs. Journal of the Experimental Analysis of Behavior, 1959, 2, 227-248.

WINOGRAD, E. Escape behavior under different fixed-ratios and shock intensities. Journal of the Experimental Analysis of Behavior, 1965, 8, 117-124.

\section{NOTES}

1. Now at North Dakota State University.

2. This research was partially supported by University Grant G-276 to M. Daley and a University research grant to J. Khalili. 